\title{
El Nuevo Consejo de Derechos Humanos de Naciones Unidas*
}

Este comentario está disponible en www.anuariocdh.uchile.cl

Morton H. Halperin $^{* *}$ y Diane F. Orentlicher ${ }^{* * *}$

E1 24 24 de marzo de 2006, la Comisión de Derechos Humanos de Naciones Unidas (la Comisión o la $\mathrm{CDH}$ ) se reunió brevemente por última vez en su sesión número 62. En una resolución de cuatro párrafos, la Comisión decidió "terminar su trabajo de conformidad" con las medidas adoptadas por la Asamblea General de la ONU nueve días antes, y remitir su trabajo pendiente al recién creado Consejo de Derechos Humanos (el Consejo) para su consideración durante la reunión inaugural en junio de $2006^{1}$.

La determinación de la Asamblea General de reemplazar a la $\mathrm{CDH}$, de 60 años de antigüedad, y sustituirla por el Consejo, evolucionó a partir de una propuesta formulada en el Informe del Grupo de Alto Nivel sobre las amenazas, los desafíos y el cambio, presentado en diciembre del 2004. Dicho Informe fue elaborado por una comisión nombrada por Kofi Annan, Secretario General de la ONU en ese momento, a la cual se encargó evaluar la mejor forma en que Naciones Unidas podía hacer frente a las actuales amenazas a la seguridad ${ }^{2}$. Como indica el Grupo de Alto Nivel, en los últimos años, la CDH había llegado a una situación generalizada de falta de legitimidad a medida que Estados con malos antecedentes en materia de derechos humanos (los que se mencionan con mayor frecuencia son Libia, Sudán, Zimbabwe y Cuba) eran elegidos como integrantes del organismo de 53 miembros. En su informe, el Grupo de Alto Nivel señala que: "Nos preocupa observar que en los últimos años ha habido Estados que se han hecho partes en la Comisión no para afianzar los derechos humanos sino para protegerse contra críticas o para criticar a otros" ${ }^{\prime \prime}$.

Dado su escepticismo frente a las propuestas de reformar la Comisión aplicando criterios más rigurosos a los miembros, el Grupo de Alto Nivel recomendó que la afiliación a la CDH se ampliara de modo de incluir a todos los Estados Miembros de Naciones Unidas ${ }^{4}$. Con miras a incrementar su profesionalismo y garantizar que los Estados no escapen al escrutinio como resul-

* Esta es una versión actualizada del artículo "The New UN Human Rights Council", publicado en inglés en Human Rights Brief, Volume 13, Issue 3 (Spring 2006), Center of Human Rights and Humanitarian Law, American University, Washington College of Law. El presente artículo fue traducido del inglés por Jennifer Ann Metcalfe.

** Director, U.S Advocacy for de Open Society Institute. Senior Fellow, Center for American Progress.

*** Professor of International Law. Co-Director, Center for Human Rights and Humanitarian Law, American University, Washington College of Law.

1 Resolución de clausura del trabajo de la Comisión (Procedural resolution on the closure of the work of the Commission), U.N. Doc. E/CN.4/2006/L.2, 2, 4 (27 de marzo de 2006), ver en http://www1.umn.edu/humanrts/closure.html (acceso efectuado el 11 de abril de 2006).

2 Informe del Grupo de Alto Nivel [nombrado por el Secretario General de la ONU] sobre las amenazas, los desafíos y el cambio en Un mundo más seguro: la responsabilidad que compartimos (2 de diciembre de 2004), ver en http://www. un.org/secureworld/ (acceso efectuado el 11 de abril de 2006).

3 Ídem, 283.

4 Ver Ídem, 285. 
tado de deliberaciones y votaciones politizadas, el Grupo de Alto Nivel también planteó que "un consejo o grupo asesor apoye a la $\mathrm{CDH}$ en su labor", el cual "constaría de unos quince expertos independientes" ${ }^{5}$. A su vez, propuso que "se pida al Alto Comisionado que prepare un informe anual sobre la situación de los derechos humanos en todo el mundo", que "podría servir de base luego para un completo debate en la Comisión" ${ }^{\prime 6}$.

En un informe publicado en marzo de 2005, titulado "Un concepto más amplio de la libertad: desarrollo, y derechos humanos para todos ${ }^{\prime 7}$, Annan apoyó varias de las reformas propuestas por el Grupo de Alto Nivel. Sin embargo, en lugar de intentar reformar la Comisión, el Secretario General propuso reemplazar el organismo desacreditado por "un Consejo de Derechos Humanos [...] de composición más reducida" ${ }^{8}$. Para realzar el prestigio del organismo al interior de las Naciones Unidas, Annan planteó que dicha entidad sea "un órgano principal de las Naciones Unidas o un órgano subsidiario de la Asamblea General" ${ }^{\prime 9}$. En contraste con lo anterior, la CDH era una entidad subsidiaria del Consejo Económico y Social (ECOSOC). El Secretario General recomendó, asimismo, que los nuevos miembros del Consejo sean "elegidos directamente por la Asamblea General por el voto de una mayoría de dos tercios de los miembros presentes y votantes"10.

La propuesta de Annan no hizo referencia alguna a la representación geográfica, una consideración informal pero crucial en la elección de los miembros de la $\mathrm{CDH}$. En cambio, el principal criterio para formar parte de la Comisión fue el compromiso del país del candidato con los derechos humanos. "Los miembros del Consejo", sostuvo Annan, "deberían comprometerse a acatar las normas de derechos humanos más rigurosas" ${ }^{11}$. Por último, como órgano permanente, el Consejo estaría en condiciones de responder cuando fuese necesario a las violaciones en curso, a diferencia de la Comisión que solamente se reunía una vez al año durante un período de seis semanas.

En un discurso pronunciado ante la CDH el 7 de abril de 2005, el Secretario General manifestó su apoyo al examen universal realizado por los pares en el nuevo Consejo, al señalar que éste:

“Debería tener una función definida especialmente para actuar como mecanismo de examen entre los países. Su misión principal sería evaluar el cumplimiento de las obligaciones relacionadas con los derechos humanos por parte de los Estados Miembros. De esta manera se concretaría el principio de universalidad e indivisibilidad de los derechos humanos"12.

En un "Plan de Acción" elaborado a partir de una solicitud planteada en "Un concepto más amplio de la libertad...", Louise Arbour, Alta Comisionada de las Naciones Unidas para los Derechos Humanos, hizo referencia al Ilamado del Secretario General de reemplazar a la Comisión por un Consejo de Derechos Humanos de mayor categoría"13, y manifestó su decidido apoyo al principio de examen universal ${ }^{14}$, así como su firme respaldo a la opinión del Secretario General de que

\footnotetext{
Ídem, 287.

6 ldem, 288.

7 Informe del Secretario General, In Larger Freedom: Towards Development, Security and Human Rights for All (21 de marzo de 2005), ver en http://www.un.org/largerfreedom/contents.htm (acceso efectuado el 11 de abril de 2006).

8 Ídem, 183.

9 Ídem.

10 Ídem.

11 ldem.

12 Discurso del Secretario General ante la Comisión de Derechos Humanos (7 de abril de 2005), ver en http://www. un.org/apps/sg/sgstats.asp?nid=1388\# (acceso efectuado el 11 de abril de 2006).

13 Ver Plan de acción presentado por la Alta Comisionada de las Naciones Unidas para los Derechos Humanos, U.N. Doc. A/59/2005/Add.3, p. 3 (26 de mayo de 2005).

14 Ídem, 92.
} 
"el nuevo Consejo de Derechos Humanos también debería continuar la práctica de la Comisión relativa al acceso de las organizaciones no gubernamentales [ONG] y mantener la función independiente de los procedimientos especiales"15.

Los "procedimientos especiales", a los que se refería la Sra. Arbour, incorporaban a relatores especiales, expertos y grupos de trabajos nombrados por la $\mathrm{CDH}$, ya fuera para abordar temas específicos, tales como la tortura y la desaparición forzosa, o temas de países cuyo historial en materia de derechos humanos es causa de particular inquietud. La opinión general es que los procedimientos especiales constituían una de las fortalezas clave de la Comisión.

De ahí hasta la reunión cumbre de la Asamblea General en septiembre de 2005, donde se reunieron los jefes de Estado para considerar propuestas de reforma de la ONU, la credibilidad del propio Secretario General se había erosionado, hecho que limitó su capacidad de obtener el respaldo necesario para su audaz visión de un Consejo de Derechos Humanos eficaz. A su vez, Naciones Unidas se había visto sacudida por una serie de escándalos, el más dañino de los cuales fue el de corrupción desenfrenada en su propio programa "petróleo por alimentos" desarrollado en Irak.

Entre tanto, en un giro algo irónico, en agosto de 2005, John Bolton, un abierto crítico de las Naciones Unidas, fue nombrado Representante Permanente de Estados Unidos ante dicha organización (el nombramiento de Bolton terminó a fines del 2006). Poco después de asumir sus nuevas funciones, él puso fin a los avanzados esfuerzos de llegar a una posición de consenso en los temas de reforma, al insistir en la realización de negociaciones directas entre los países. La postura de la delegación de EE.UU. fue un factor clave en la reducción drástica de la esperanza de reformar a la ONU en varios aspectos, entre ellos la creación del nuevo Consejo de Derechos Humanos.

El 20 de septiembre de 2005, cuando los jefes de Estado aprobaron una declaración de consenso, lo único que aún permanecía del texto anterior sobre el nuevo Consejo -mayormente acordado-, era el encargo al Presidente de la Asamblea General, que en ese entonces ocupaba el diplomático sueco Jan Eliasson, de lograr que dicha Asamblea aprobara de manera transparente una resolución que creara el nuevo Consejo de Derechos Humanos. Habían desaparecido una serie de concesiones otorgadas por gobiernos abusivos a cambio de la terminología que deseaban que se utilizara frente a otros temas.

Tratando de salvar la situación, Eliasson nombró a los Representantes Permanentes de Sudáfrica y Panamá para que llevaran a cabo las negociaciones en su representación. Durante los meses siguientes, predominaron tres temas en las negociaciones: cómo se elegirían los miembros del nuevo Consejo; con qué frecuencia se reuniría el Consejo, y cómo haría frente a asuntos específicos relacionados con cada país. Fue relativamente fácil llegar a un consenso sobre los dos últimos puntos. En lo que se refiere a las reuniones del Consejo, la Resolución 60/251 de la Asamblea General, que determina las condiciones de operación del Consejo, estableció que:

[...] el Consejo se reunirá periódicamente a lo largo del año y celebrará como mínimo tres períodos de sesiones por año, incluido un período de sesiones principal, que tendrán una duración total no inferior a diez semanas, y podrá celebrar períodos extraordinarios de sesiones, cuando sea necesario, a solicitud de un miembro del Consejo ${ }^{16}$.

En cuanto a asuntos específicos relacionados con cada país, aunque la Asamblea General impartió instrucciones al nuevo Consejo para que realizara "un examen periódico universal ... sobre el cumplimiento por parte de cada Estado de sus obligaciones y compromisos en materia de derechos

\footnotetext{
15 Ídem.

16 U.N. Doc. A/RES/60/251, 10 (3 de abril de 2006).
} 
humanos de manera que garantice la universalidad del examen y la igualdad de trato respecto de todos los Estados"17, dejó en claro que el Consejo también puede identificar a quienes llevan a cabo violaciones graves de los derechos humanos y someterlos a un examen especial. ${ }^{18}$

Asimismo, se desarrolló terminología de amplia aceptación para garantizar que las ONG tuvieran, básicamente, el mismo grado de acceso al Consejo que tenían durante la vigencia de la Comisión ${ }^{19}$; para conservar el papel de los procedimientos especiales, así como para examinar y, "cuando sea necesario", perfeccionar y racionalizar "todos los mandatos, mecanismos, funciones y responsabilidades" de la $\mathrm{CDH}^{20}$.

Asimismo, al determinar que el Consejo deberá formular "recomendaciones a la Asamblea General para seguir desarrollando el derecho internacional en la esfera de los derechos humanos" ${ }^{21}$, la resolución intentó garantizar que el Consejo siguiera haciendo prosperar uno de los éxitos singulares de la $\mathrm{CDH}$, a saber, el desarrollo de normas eficaces de derechos humanos. Efectivamente, mucho antes de que la Comisión desarrollara sus instrumentos de implementación, se ganó el respeto a escala mundial por el papel que desempeñó en el desarrollo de normas. Como señalara la Alta Comisionada para los Derechos Humanos en la sesión final de la CDH, los instrumentos que conforman la Declaración Universal de los Derechos Humanos, que fueron desarrollados por la Comisión en las primeras etapas de su historia, "fueron y siguen siendo instrumentos pioneros, quizás la contribución más famosa hecha por las Naciones Unidas al bienestar de toda la humanidad"22.

Aunque fue relativamente fácil lograr un consenso sobre el papel de fijación de normas del Consejo, el tema de los criterios y procesos de elección de los nuevos miembros continuó siendo polémico hasta el fin del proceso de negociación en Nueva York. Durante la reunión cumbre, pronto quedó en evidencia que la idea de abandonar el criterio de representación geográfica contaba con muy poco apoyo ${ }^{23}$. También resultaba claro que el paquete de reformas subsanaría un desequilibrio existente en la representación en la $\mathrm{CDH}$, vale decir, que un número excesivo de puestos eran ocupados por países pertenecientes al Grupo de Estados de Europa Occidental y otros y por el Grupo de América Latina y el Caribe ${ }^{24}$.

A pesar de que existía un acuerdo generalizado de que los miembros del Consejo debían ser elegidos por la Asamblea General (en lugar del ECOSOC), no se logró apoyo para llevar adelante una reforma a la Carta de las Naciones Unidas destinada a crear un nuevo cuerpo primario. En consecuencia, el nuevo Consejo es un órgano subsidiario de la Asamblea General ${ }^{25}$.

\footnotetext{
17 Ídem, 5(e).

18 Ver Ídem, 3 (la Asamblea General "Decide también que el Consejo deberá ocuparse de las situaciones en que se violen los derechos humanos, incluidas las violaciones graves y sistemáticas, y hacer recomendaciones al respecto").

19 Ídem, 11. Sin embargo, en una redacción que causó inquietud a muchas ONG, la resolución agrega que el Consejo deberá asegurar "la contribución más eficaz posible de esas entidades", entre varias otras.

20 Ídem, 6.

21 Ídem, 5(c).

22 Louise Arbour, Alta Comisionada de Naciones Unidas para los Derechos Humanos, On the Occasion of the 62nd session of the Commission on Human Rights, p. 4 (27 de marzo de 2006), ver en http://www.treatycouncil.org/PDFs/ HC.OPENING.FINAL.pdf (acceso efectuado el 11 de abril de 2006).

23 La Resolución 60/251 de la Asamblea General estableció que: "la composición [del Consejo] estará basada en una distribución geográfica equitativa". UN Doc. A/RES/60/251, 7.

24 ver Ídem.

25 Ídem, 1.
} 
El debate más intenso se centró en los procedimientos de elección. Estados Unidos, la Unión Europea (UE) y los países que compartían su propuesta propugnaban por un proceso que impidiera la elección de graves violadores de derechos humanos, pero no lograban ponerse de acuerdo respecto de los diferentes procedimientos posibles. Estados Unidos presionaba a favor de una disposición que vedara el acceso a los países sancionados por el Consejo de Seguridad de Naciones Unidas por violaciones a los derechos humanos o por apoyar al terrorismo. La Unión Europea promovía una propuesta conforme a la cual cada bloque regional presentara un número de países mayor que la cantidad de puestos asignados a la región, para garantizar así que la Asamblea General pudiera hacer una elección significativa, excluyendo de ese modo a los peores transgresores.

Estados Unidos respaldó la propuesta del Secretario General formulada en "Un concepto más amplio de la libertad", conforme a la cual la Asamblea General elegiría a los miembros del Consejo mediante dos tercios de los votos. Esta propuesta resultaba atractiva, en parte porque la había formulado el Secretario General en lugar del bloque occidental, pero también tenía varios opositores. Algunos Estados con trayectorias negativas en el área de derechos humanos, como Cuba, Egipto y Argelia, temían que el límite de los dos tercios les impediría tener acceso al Consejo. Se afirma también que a algunos funcionarios del gobierno de EE.UU. les preocupaba que, a la luz de la crítica internacional frente a su política de detenciones implementada en Guantánamo y otros lugares, su práctica de la "rendición extraordinaria" de los sospechosos de actos de terrorismo y los abusos cometidos por sus fuerzas militares en el campo de detenidos de Abu Ghraib y en otros sitios, Estados Unidos no lograra obtener los dos tercios de respaldo de los miembros de Naciones Unidas.

En febrero de 2006, el Presidente de la Asamblea General presentó un borrador que contenía una fórmula intermedia, el cual fue aprobado por ésta el 15 de marzo de 2006. El nuevo Consejo tendría 47 miembros, "elegidos en forma directa e individual en votación secreta por la mayoría de los miembros de la Asamblea General"26. La participación en el Consejo, a diferencia de la $\mathrm{CDH}$, estaría sujeta explícitamente a criterios de derechos humanos: al elegir a los miembros del Consejo, los Estados Miembros de Naciones Unidas "deberán tener en cuenta la contribución de los candidatos a la promoción y protección de los derechos humanos y las promesas y compromisos voluntarios que hayan hecho al respecto" ${ }^{\prime 27}$. Los Estados elegidos como miembros del Consejo deberán (al menos en principio) "aplicar las normas más estrictas en la promoción y protección de los derechos humanos, cooperar plenamente con el Consejo y estarán sujetos al mecanismo de examen periódico universal durante el período en que sean miembros" ${ }^{28}$. Además, la Asamblea General "por mayoría de los miembros presentes y votantes, podrá suspender los derechos inherentes a formar parte del Consejo de todo miembro de éste que cometa violaciones graves y sistemáticas de los derechos humanos" 29 .

La mayoría de los Estados concordaron con el Presidente Eliasson en cuanto a que esta redacción era la mejor posible, dada la atmósfera existente y que era urgente consolidar el acuerdo antes de que se realizara la siguiente reunión de la CDH. Estados Unidos no compartía esta opinión. El Embajador Bolton manifestó su inquietud acerca de muchas disposiciones contenidas en el texto y, junto con negarse a explicitar cuáles cambios lograrían que Estados Unidos apoyara la resolución, hizo un nuevo llamado a efectuar negociaciones individuales entre países. El apoyo para esta posición fue casi nulo y finalmente la Asamblea aprobó la resolución que creaba el nuevo

\footnotetext{
26 Ídem, 7.

27 Ídem, 8.

28 ídem, 9.

29 Ídem.
} 
Consejo por una votación de 170 contra 4, en la cual sólo EE.UU., Israel, Palau y las Islas Marshall se opusieron, mientras que otros tres países (Irán, Bielorrusia y Venezuela) se abstuvieron.

Luego de la votación, numerosas ONG y países con ideas afines lanzaron una iniciativa para convencer a los Estados con un historial positivo en materia de derechos humanos para que postularan al Consejo, solicitándoles el compromiso de no votar por países con una mala trayectoria en esta materia. El 6 de abril de 2006, Estados Unidos anunció que no se presentaría a la elección de la primera sesión del Consejo pero que respaldaría a los países que considerara dignos de ser miembros ${ }^{30}$.

\section{El Consejo de Derechos Humanos en acción}

Producto de las buenas intenciones pero creado en circunstancias imperfectas, el inicio del Consejo fue decepcionante. A pesar de los nuevos criterios y procedimientos implementados para las elecciones con miras a mantener fuera del órgano a los violadores flagrantes de derechos humanos, algunos países que sistemáticamente violan los derechos humanos, tales como Argelia, Túnez, Cuba y Arabia Saudita, fueron elegidos al primer Consejo. Dirigidos por Argelia y Pakistán, estos países han presionado con éxito para que la atención se enfoque principalmente en Israel y han logrado resistir resoluciones relacionadas con otras situaciones graves que afectan a los países. Además, trataron de reducir el papel que desempeñan las ONG en las deliberaciones del Consejo.

Sin perjuicio de lo anterior, los actuales integrantes del Consejo representan un adelanto en comparación con la $\mathrm{CDH}$. Algunos Estados cuya participación en la $\mathrm{CDH}$ había empañado su reputación, entre los que se cuentan Irán, Venezuela, Zimbabwe, Sudán, Libia, Egipto y Siria, no fueron miembros del Consejo durante su primer año de funcionamiento. Del total de 47 miembros elegidos originalmente, 37 provenían de la Comunidad de Democracias, una coalición de estados cuyo objetivo es promover y fortalecer la gestión democrática. En contraste, durante la última sesión de la Comisión, solamente 33 de sus 53 miembros eran miembros de dicha Comunidad.

Durante su primera sesión regular, el Consejo adoptó los textos de dos importantes instrumentos de derechos humanos. La primera resolución del Consejo, del 29 de junio de 2006, aprobó la Convención Internacional para la Protección de Todas las Formas de Desaparición Forzada ${ }^{31}$, la cual fue posteriormente aprobada por la Asamblea General el 20 de diciembre de $2006^{32}$ y abierta para las firmas el 6 de febrero de 2007. También el 29 de junio de 2006, el Consejo adoptó un texto preliminar de Declaración sobre los Derechos de los Pueblos Indígenas ${ }^{33}$.

No obstante, mientras la primera sesión regular del Consejo legó dos hitos para el mayor desarrollo de los estándares en materia de derechos humanos, su mayoría democrática todavía tiene que lograr un liderazgo eficaz con respecto a cuestiones específicas en diferentes países. Esta falencia continúa siendo un factor crucial en el desempeño decepcionante que ha demostrado la entidad hasta la fecha. En momentos en que la situación de los derechos humanos en Darfur, Sudán y en otras partes del mundo requería atención urgente, el Consejo dedicó sus tres primeros períodos de sesiones exclusivamente a la situación de los derechos humanos en Palestina y Líbano. El resultado de estas reuniones, realizadas en julio, agosto y noviembre de 2006, fueron

\footnotetext{
30 Ver Warren Hoge, N.Y. Times, "U.S. Says It Won't Be Candidate for U.N. Rights Council" (6 de abril de 2006).

31 Resolución 1/1 del Consejo de Derechos Humanos, 29 de junio de 2006.

32 GA Res. 61/177, UN Doc. A/RES/61/177 (20 Dec. 2006). La Asamblea General había aplazado la consideración de esta Convención para su Sesión $N^{\circ}$ 61, en la cual finalmente la aprobó por unanimidad.

33 Resolución 1/2 del Consejo de Derechos Humanos, 29 de junio de 2006.
} 
resoluciones que condenaban las violaciones a los derechos humanos cometidas por Israel, pero no así las ejecutadas por otros. Por ejemplo, la resolución aprobada durante el primer período extraordinario de sesiones del Consejo expresa "grave preocupación" por las violaciones de los derechos humanos del pueblo palestino causadas por la ocupación israelí y hace un Ilamamiento a Israel a poner fin a una amplia gama de prácticas realizadas en el territorio palestino ocupado. No obstante, mientras el Consejo instaba a "todas las partes interesadas" a que respetasen las normas del derecho internacional humanitario, no hacía mención alguna de los actos terroristas realizados por grupos armados palestinos ${ }^{34}$. Durante su segundo período extraordinario de sesiones, el Consejo creó una Misión de Investigación para que examinara las actividades militares israelíes en Líbano, pero la Misión no contaba con autorización para investigar las violaciones cometidas por Hezbollah ${ }^{35}$. La Unión Europea y los estados con ideas afines se opusieron a estos esfuerzos pero se vieron superados por las tácticas de un bloque bien organizado, conducido por Argelia en su papel de dirigente de la Organización de la Conferencia Islámica.

Human Rights Watch, que documentó las violaciones a los derechos humanos y al derecho humanitario tanto por parte de Israel como de Hezbollah durante la guerra de 2006 entre Israel y Líbano, condenó "el enfoque unilateral adoptado por el Consejo de Derechos Humanos" en su sesión extraordinaria de agosto de 2006, a la vez que lo catalogó como "un golpe a la credibilidad [del Consejo] y una renuncia a su responsabilidad de proteger los derechos humanos de todos" ${ }^{\prime \prime 6}$.

Al comentar sobre los primeros años del Consejo, a mediados de diciembre de 2006, Kofi Annan, el saliente Secretario General, señaló que el Consejo "claramente no ha justificado las esperanzas" depositadas en él. En una crítica a la entidad por su "atención desproporcionada a las violaciones cometidas por Israel", Annan agregó: "Esto no significa que debiera darse libertad de acción a Israel .... Pero el Consejo debiera prestar la misma atención a las graves violaciones cometidas por otros estados" 37 .

No fue sino hasta mediados de diciembre de 2006, unos seis meses después de iniciado su trabajo, que el Consejo finalmente convocó a un período extraordinario de sesiones para abordar la crisis de Darfur. Esto se produjo a instancias de la Unión Europea y de Estados con ideas afines, y representó un esfuerzo concertado y respaldado por el gobierno de EE.UU. con el fin de quitarles el liderazgo del Consejo a los países abusivos. En particular, las ONG del sur desempeñaron un papel importante en presionar a los gobiernos para que apoyaran el período extraordinario de sesiones y lograr una firme resolución sobre Darfur. En la práctica, la resolución del Consejo no condenó específicamente al gobierno de Sudán pero acordó enviar un grupo de expertos a Darfur.

A medida que transcurría el primer año del Consejo y las consecuentes dificultades iniciales, gran parte de las deliberaciones de su período normal de sesiones fueron dedicadas a aspectos de procedimiento y a cuestiones operativas. En su primer período normal de sesiones (junio de 2006), el Consejo estableció un grupo de trabajo intergubernamental para funcionar entre los períodos de sesiones con el fin de desarrollar las modalidades del proceso periódico de examen universal. Un tema clave a tratar por este grupo era si participarían expertos independientes en el

\footnotetext{
34 Consejo de Derechos Humanos, Sesión Especial, Resolución S-1/1, Human Rights Situation in the Occupied Palestinian Territory, 1, 5; ver en http://www.ohcr CHR.org/english/bodies/hrcouncil/docs/specialsession/A.HRC.RES. S-1.1_en.pdf?si=A/HRC/S-1/1.

35 "Report of the Commission of Inquiry on Lebanon pursuant to Human Rights Council resolution S-2/1", 1, 5-7, ver en http://www.ohla CHR.org/english/bodies/hrcouncil/docs/specialsession/A.HRC.3.2.pdf.

36 Human Rights Watch, Human Rights News, 11 de agosto de 2006. "Lebanon/Israel: U.N. Rights Body Squanders Chance to Help Civilians", ver en http://hrw.org/english/docs/2006/08/11/lebano13969.htm

37 "Candor from the departing Annan", Chicago Daily Law Bulletin, 15 de diciembre de 2006.
} 
proceso de examen universal. A su vez, en la misma sesión, el Consejo creó otro grupo de trabajo con el fin de que formulara recomendaciones para el examen, mejoramiento y racionalización del sistema de procedimientos especiales mientras que, simultáneamente, amplió por un año los mandatos de los procedimientos especiales establecidos. Entre los temas clave que debía abordar este grupo de trabajo se incluyen la decisión sobre la continuidad de los mandatos específicos relacionados con países en vista del sistema de examen universal y la determinación del proceso para nombrar a los mandatarios. Estos asuntos serán sometidos a votación en junio de 2007.

En resumen, durante su primera sesión regular el Consejo ha continuado la reconocida tradición de la $\mathrm{CDH}$ en la determinación de estándares en materia de derechos humanos, adoptando dos instrumentos claves sobre desaparición forzada y derechos de los pueblos indígenas. A pesar de eso, la nueva entidad ya se ha visto empañada por la trayectoria que ostentan algunos de sus miembros en el campo de los derechos humanos; su dedicación exclusiva a los abusos de Israel durante sus primeros tres períodos de sesiones extraordinarias, y su incapacidad para responder adecuadamente a otras situaciones que requerían atención urgente. Sin perjuicio de su decepcionante desempeño sobre algunas cuestiones durante los primeros meses, es demasiado pronto para afirmar que el Consejo es un fracaso. La sesión especial sobre Darfur realizada en diciembre de 2006 da motivos para tener esperanzas de que la entidad aún pueda llegar a constituirse en un organismo mejor que la desacreditada Comisión. Otras pruebas cruciales se producirán en junio de 2007, fecha en que está programado que el Consejo apruebe resoluciones sobre procedimientos especiales y el examen universal periódico. Mientras tanto, deberá renovarse un tercio de los escaños de la entidad en mayo de 2007, ocasión propicia para mejorar su composición. Sin embargo, ninguna de estas oportunidades tendrá éxito para mejorarlo a menos que los Estados comprometidos con los derechos humanos desempeñen un papel de mayor liderazgo que el ejercido hasta ahora. 